\title{
A Joint Perspective Towards Image Super-Resolution: Unifying External- and Self-Examples
}

\author{
Zhangyang Wang $\dagger$, Zhaowen Wang $\dagger$, Shiyu Chang $\dagger$, Jianchao Yang $\ddagger$, and Thomas Huang $\dagger$ \\ $\dagger$ †eckman Institute, University of Illinois at Urbana-Champaign, Urbana, IL 61801, USA \\ $\ddagger$ Adobe Systems Inc, San Jose, CA 95110, USA \\ \{zwang119, wang308, chang87\}@illinois.edu, jiayang@adobe.com, huang@ifp.uiuc.edu
}

\begin{abstract}
Existing example-based super resolution (SR) methods are built upon either external-examples or self-examples. Although effective in certain cases, both methods suffer from their inherent limitation. This paper goes beyond these two classes of most common example-based SR approaches, and proposes a novel joint SR perspective. The joint $S R$ exploits and maximizes the complementary advantages of external-and self-example based methods. We elaborate on exploitable priors for image components of different nature, and formulate their corresponding loss functions mathematically. Equipped with that, we construct a unified SR formulation, and propose an iterative joint super resolution (IJSR) algorithm to solve the optimization. Such a joint perspective approach leads to an impressive improvement of $S R$ results both quantitatively and qualitatively.
\end{abstract}

\section{Introduction}

Super-resolution (SR) algorithms aim to construct a high-resolution (HR) image from one or multiple lowresolution (LR) input frames [1]. However, this problem is essentially ill-posed because the ground truth is unknown, and much information is lost in the HR-toLR degradation process. Existing SR algorithms can be broadly categorized into three classes: interpolation-based [?, 2], reconstruction-based [3], and example-based methods $[6,8]$. The former two suffer from producing oversmoothed images with much detail loss. In addition, their performances often degrade rapidly with increasing scaling factors [3].

The current most popular single image SR methods rely on example-based learning techniques, which attempt to learn priors for reconstructing HR images from a set of training examples. Classical example-based methods learn the mapping between LR and HR image patches from a large and representative set of image pairs, and then apply

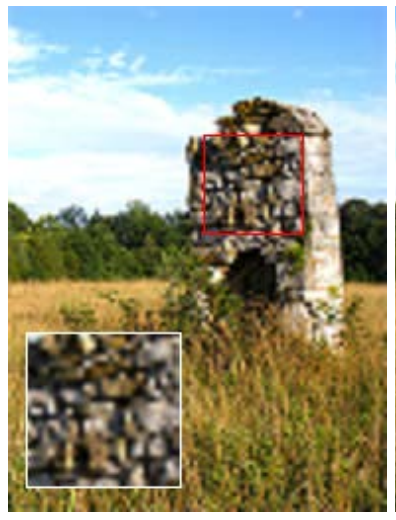

(a)

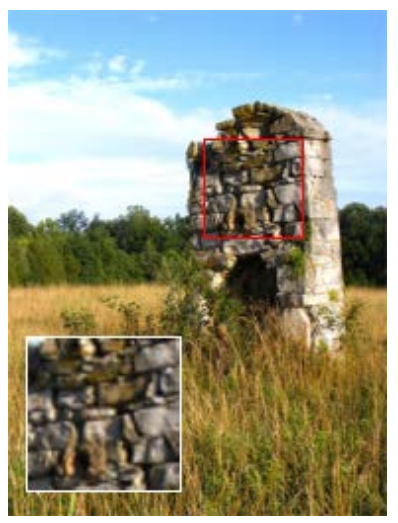

(b)
Figure 1. The SR result of "Wall” image with a magnification factor 3, using bicubic interpolation (a) and our method (b).

the learned mapping to SR $[4,5,6]$. Since the examples are trained from an external image set, we denote such kind of methods as "external-example based". More recently, there also emerges work stressing on exploiting self-similar examples for SR using only the single input frame as references $[8,9]$. As their examples are learned from the input image itself, we denote such kind of methods as "selfexample based". Although either external or self-example based methods show nice SR results on some images, there still exist inherent limitations on their performances, which we will reveal by a comparison study in Section 2 .

In this paper, we propose a novel joint SR perspective instead of focusing on exploiting either external-examples or self-examples alone. Fig. 1 shows an impressive result of the proposed method in successfully reconstructing very complicated structures and textures, e.g., the brick regions, without introducing noticeable visual artifact. To carry out the joint SR, we make multifold efforts and contributions in this paper. We are the first to formulate a joint SR model, which enables the interaction between the effects of external and self examples through their respective effective priors. The model lays its foundation on an in-depth study 
towards the complementary inherent properties in externaland self-example based viewpoints. In order to solve the formulated SR model, we design an iterative joint super resolution (IJSR) algorithm. Experimental results confirm the superiority of the new method in both quantitative and qualitative comparisons.

\section{Related Work}

Let $\mathbf{X}$ denote the HR image to be estimated from the LR input $\mathbf{Y} . \mathbf{X}_{i j}$ and $\mathbf{Y}_{i j}$ stand for the $(i, j)$-th $(i, j=1,2 \ldots)$ patch from $\mathbf{X}$ and $\mathbf{Y}$, respectively.

\subsection{External-Example based Methods and Generic Prior}

External-example based SR methods use a universal set of example patches to train dictionaries for predicting the missing (high frequency) information for the HR image. The basic assumption they follow is concluded as:

Generic Prior: A patch of an input image often recurs or finds its similar counterpart in many other natural images, and can thus be compactly represented by a pre-trained universal dictionary.

A popular class of external-example based SR methods are associated with the sparse coding technique [12,5]. It is stated by the sparse coding prior that the patches of a natural image can be represented as a sparse linear combination of elements in a redundant pre-trained dictionary. For image SR problems, an advanced coupled sparse coding prior is adopted in some state-of-the-art SR methods [5, 6]. The HR and LR patch spaces $\left\{\mathbf{X}_{i j}\right\}$ and $\left\{\mathbf{Y}_{i j}\right\}$ are actually tied by some mapping function. With a well-trained coupled dictionary pair $\left(D_{h}, D_{l}\right)$ (please see [5, 6] for details), the coupled sparse coding prior [5] concludes that $\left(\mathbf{X}_{i j}, \mathbf{Y}_{i j}\right)$ tends to admit a joint sparse representation $\mathbf{a}_{i j}$ :

$$
\begin{array}{ll}
\min _{\mathbf{X},\left\{\mathbf{a}_{i j}\right\}} & \sum_{\{i, j\}, \mathbf{X}_{i j} \in \mathbf{X}}\left\|\mathbf{a}_{i j}\right\|_{1}+\lambda_{l}\left\|D_{l} \mathbf{a}_{i j}-\mathbf{Y}_{i j}\right\|_{2}^{2} \\
& +\lambda_{h}\left\|D_{h} \mathbf{a}_{i j}-\mathbf{X}_{i j}\right\|_{2}^{2}
\end{array}
$$

However in practice, since $\mathbf{X}$ is unknown, the true $\mathbf{a}_{i j}$ is also unavailable. The authors in [5] suggest to first infer the sparse representation $\mathbf{a}_{i j}^{L}$ of $\mathbf{Y}_{i j}$ with respect to $D_{l}$, by minimizing (2):

$$
\min _{\mathbf{X},\left\{\mathbf{a}_{i j}^{L}\right\}} \sum_{\{i, j\}, \mathbf{X}_{i j} \in \mathbf{X}}\left\|\mathbf{a}_{i j}^{L}\right\|_{1}+\lambda_{l}\left\|D_{l} \mathbf{a}_{i j}^{L}-\mathbf{Y}_{i j}\right\|_{2}^{2}
$$

and then use $\mathbf{a}_{i j}^{L}$ as an approximation of $\mathbf{a}_{i j}^{H}$ (the sparse code of $\mathbf{X}_{i j}$ with respect to $D_{h}$ ), to recover $\mathbf{X}_{i j} \approx D_{h} \mathbf{a}_{i j}^{L}$.

External-example based methods are capable of producing plausible fine details and sharpened edges. However, because textures contained in the input image may not have any counterparts in the universal external database, most external-example based methods are prone to produce noise and irregularities along curved edges or textured areas [9]. With any finite-sized external training set, it is impossible to represent all the image patterns, especially those uncommon textures. It implies that though reasonable for most parts, there do exist regions in an input image that the generic prior cannot apply. One possible solution is to construct a larger data set to encompass as many image variations as possible. However, it seems almost impossible to include all kinds of singular features [10], and the use of a huge database will ensue unaffordable computational load. Dong et al. [13] exploits the image nonlocal self-similarity to refine the estimates of the sparse coding coefficients, but their results are accompanied with the oversmoothed details.

\subsection{Self-Example based Methods and Individual Prior}

Self-example based methods suggest the utility of input image at multiple scales as the source of examples. Their effectiveness hinges on the following prior of natural images:

Individual Prior: A patch of an input image can find its relevant example patches from the input itself, within the same scale and across different scales.

Although self-examples provide a limited number of references, they are very relevant to the input image and thus shown to be effective for SR [7, 11, 9]. Particularly, Freedman and Fattal [8] observed that small patches, especially singular features like edges and corners, tend to repeat almost identically across different image scales (under small scaling factors). Such local scale invariance prior allows the algorithm to search relevant example patches efficiently in very restricted neighborhoods around the same relative coordinates at multiple scales of the input image.

Define a linear interpolation operator $\mathcal{U}$ (with scaling factor $s$ ) and a downsampling operator $\mathcal{D}$ (with scaling factor $\frac{1}{s}$ ), for the input LR image $\mathbf{Y}$, we first obtain its initial upsampled image $\mathbf{X}^{\prime}=\mathcal{U}(\mathbf{Y})$, and a smoothed input image $\mathbf{Y}^{\prime}=\mathcal{U}(\mathcal{D}(\mathbf{Y}))$. Given the smoothed patch $\mathbf{X}^{\prime}{ }_{i j}$, Freedman and Fattal [10] predict the missing highfrequency band of each unknown $\mathbf{X}_{i j}$, by first solving the following minimization (3):

$$
\left(m^{*}, n^{*}\right)=\arg \min _{(m, n) \in \mathcal{W}_{i j}}\left\|Y_{m n}^{\prime}-X_{i j}^{\prime}\right\|_{2}^{2},
$$

where $\mathcal{W}_{i j}$ is defined as a small searching window (e.g., $10 \times 10)$ centered at $\left(\frac{i}{s}, \frac{j}{s}\right)$ on image $\mathbf{Y}^{\prime}$. They then get the co-located patch $\mathbf{Y}_{m^{*} n^{*}}$ from $\mathbf{Y}$. The predicted highfrequency band of each $\mathbf{X}_{i j}, \mathbf{H}\left(\mathbf{X}_{i j}\right)=\mathbf{Y}_{m^{*} n^{*}}-\mathbf{Y}_{m^{*} n^{*}}$, is used to fill-in $\mathbf{X}^{\prime}{ }_{i j}$ by simply pasting it, i.e., $\mathbf{X}_{i j}=\mathbf{X}_{i j}^{\prime}+$ $\mathbf{H}\left(\mathbf{X}_{i j}\right)$.

In contrast to methods based on external examples, selfexample based methods perform especially well in reconstructing details that are unique to the input image. However, their effects are less satisfactory on patches with- 
out self-similarity property. As observed empirically and shown by some experimental results [8], some regions in an input image cannot be well approximated by copying highfrequency information from one or more self-examples. Due to the unavailability of sufficient patch pairs, the mismatches of self-examples are difficult to be self-corrected, which introduce artifacts in images cluttered with fine details.

\section{A Joint Perspective Towards SR}

As mentioned above, the external-example and selfexample based methods both have pros and cons. However, their unique characteristics imply that superior SR results can be expected via a proper integration of the two:

- On one hand, the external-example based approach generates visually pleasant SR results for a large portion of the image. But it sometimes leads to artifacts for singular features that cannot be well represented by a pre-trained dictionary. In this case, we can resort to the self-example based approach to restore those unique details.

- On the other hand, when a patch cannot find any proper self-examples in the input image as its high frequency reference, it is a better choice to adopt sparse coding over the external dictionary, which usually leads to a smoother and natural-looking enlarged area.

This fact inspires us to develop a joint perspective that integrates the two methodologies for SR. Concretely, we propose the "Unified Prior" for SR stated as below.

Unified Prior: The set of all patch indexes $(i, j)$ in an input image, denoted as $\Omega$, can be partitioned into two mutually exclusive subsets. One subset $\mathcal{G}$, termed as "Generic Feature Component", includes patches that are suitable to apply generic prior for SR. The other subset $\mathcal{I}$, termed as "Individual Feature Component" in contrast, refers to patches where individual prior is a better choice. $\mathcal{G}$ and $\mathcal{I}$ satisfy $\mathcal{G} \cup \mathcal{I}=\Omega$ and $\mathcal{G} \bigcap \mathcal{I}=\emptyset$.

We define two loss functions $\ell_{\mathcal{G}}(\cdot)$ and $\ell_{\mathcal{I}}(\cdot)$ that enforce the generic and individual prior for regularization, respectively. All patches in $\mathbf{X}$ are classified into two disjoint sets: the set of generic feature patches $P_{\mathcal{G}}(\mathbf{X})$, and the set of individual feature patches $P_{\mathcal{I}}(\mathbf{X})$. The projector $P_{\mathcal{G}}$ is defined as: $P_{\mathcal{G}}(X)=\left\{X_{i j} \mid(i, j) \in \mathcal{G}\right\}$, and $P_{\mathcal{I}}$ is similarly defined. With these notations, we express our unified formulation towards SR in the following form:

$$
\min _{\mathbf{X}} \ell_{\mathcal{G}}\left(P_{\mathcal{G}}(\mathbf{X})\right)+\alpha \ell_{\mathcal{I}}\left(P_{\mathcal{I}}(\mathbf{X})\right)
$$

where $\alpha$ balances the two loss terms. The formulation (4) integrates priors from both external- and self-example based viewpoints, and is driven by minimizing a weighted combination of the two loss functions.
Two key issues remain to be resolved here: 1) how to design the concrete forms of functions $\ell_{\mathcal{G}}(\cdot)$ and $\left.\ell_{\mathcal{I}}(\cdot) ; 2\right)$ how to determine the two sets $\mathcal{G}$ and $\mathcal{I}$. We will address the first issue right here, while explaining the second one in Section 4.

\subsection{Symmetric Sparse Coding Prior}

In (2), note that $\mathbf{a}_{i j}$ is only optimal in the concatenated feature space of $\left\{\mathbf{X}_{i j}\right\}$ and $\left\{\mathbf{Y}_{i j}\right\}$, but not in each feature space individually. It is hence not guaranteed that $\mathbf{a}_{i j}^{H}$ is consistent with $\mathbf{a}_{i j}^{L}$. Such an optimization strategy is "asymmetric" over $\mathbf{a}_{i j}^{H}$ and $\mathbf{a}_{i j}^{L}$, since the former is totally determined by the latter and there is no information exchange/sharing between HR and LR patch spaces.

In contrast, we adopt a "symmetric" coupled sparse coding prior to design $\ell_{\mathcal{G}}$ function. We hope to explicitly include both $\left\{\mathbf{a}_{i j}^{H}\right\}$ and $\left\{\mathbf{a}_{i j}^{L}\right\}$ as optimization variables, with their corresponding sparse coding priors, while enforcing the two to be approximately equivalent. Moreover, this prior will work on $P_{\mathcal{G}}(\mathbf{X})$ rather than determining the entire $\mathbf{X}$. Therefore, we define the following function:

$$
\begin{aligned}
& \ell_{\mathcal{G}}\left(P_{\mathcal{G}}(\mathbf{X}),\left\{\mathbf{a}_{i j}^{H}\right\},\left\{\mathbf{a}_{i j}^{L}\right\}\right)=\sum_{\{i, j\} \in \mathcal{G}} \\
& \left(\left\|\mathbf{a}_{i j}^{H}\right\|_{1}+\left\|\mathbf{a}_{i j}^{L}\right\|_{1}\right)+\lambda_{l}\left\|D_{l} \mathbf{a}_{i j}^{L}-\mathbf{Y}_{i j}\right\|_{2}^{2} \\
& +\lambda_{h}\left\|D_{h} \mathbf{a}_{i j}^{H}-\mathbf{X}_{i j}\right\|_{2}^{2}+\theta\left\|\mathbf{a}_{i j}^{H}-\mathbf{a}_{i j}^{L}\right\|_{2}^{2}
\end{aligned}
$$

"Symmetric" means that in our formulation, $\mathbf{a}_{i j}^{H}$ and $\mathbf{a}_{i j}^{L}$ are equally treated (namely the constraints on $\mathbf{a}_{i j}^{H}$ and $\mathbf{a}_{i j}^{L}$ are of the same form), which is in contrast to the asymmetrical coupled sparse coding (2) where $\mathbf{a}_{i j}^{H}$ is totally determined by $\mathbf{a}_{i j}^{L}$.

\subsection{Local Scale Invariance Prior}

In our proposed scheme, instead of applying the local scale invariance prior [8] to the entire $\mathbf{X}$, we utilize it for reconstructing the $P_{\mathcal{I}}(\mathbf{X})$ part. What's more, as directly copying high-frequency part from one patch to another easily introduces rigid artifacts, especially in detailed cluttered regions, we would also like to relax the constraint, to make the recovered $\mathbf{X}_{i j}$ close (rather than exactly the same) to the prediction from self examples. The $\ell_{\mathcal{I}}(\cdot)$ function is then designed to reflect such a relaxation of the local scale invariance prior:

$$
\begin{aligned}
& \ell_{\mathcal{I}}\left(P_{\mathcal{I}}(\mathbf{X})\right)=\sum_{\{i, j\} \in \mathcal{I}} \\
& \left\|\mathbf{X}_{i j}^{\prime}+\mathbf{Y}_{m^{*} n^{*}}-\mathbf{Y}_{m^{*} n^{*}}^{\prime}-\mathbf{X}_{i j}\right\|_{2}^{2}
\end{aligned}
$$

\section{An Iterative Joint Super Resolution (IJSR) Algorithm}

Directly solving (4) is very complex due to the its high nonlinearity and entanglement among all variables 


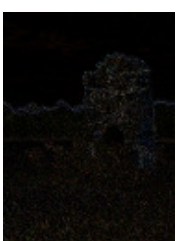

(a)

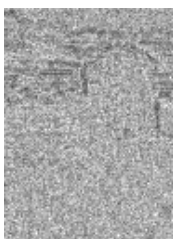

(b)

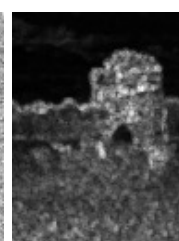

(c)

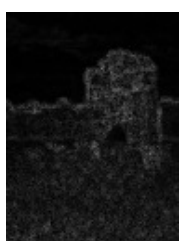

(d)

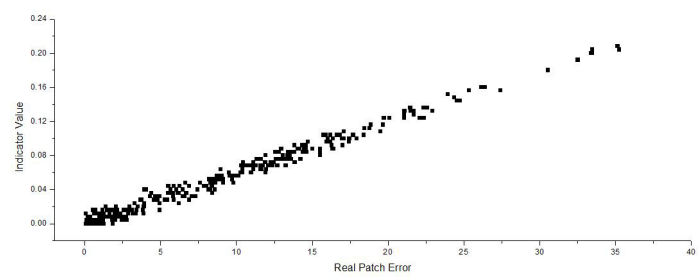

(e)

Figure 2. Validation of our proposed classifier $\mathbf{T}\left(\mathbf{a}_{i j}^{H}, \mathbf{a}_{i j}^{L}\right)$. (a) the patch-level error map; (b) the sparsity map; (c) the residual map; (d) the indicator value map; (e) shows the co-linearity of patch errors and indicator values.

$\left\{\mathbf{X},\left\{\mathbf{a}_{i j}^{H}\right\},\left\{\mathbf{a}_{i j}^{L}\right\}\right\}$. Moreover, the classification of $\mathcal{G}$ and $\mathcal{I}$ is difficult to be determined in advance as the groundtruth $\mathbf{X}$ is unknown. Instead, we choose to iteratively adjust $\mathcal{G}$ and $\mathcal{I}$ within the optimization procedure as well. In each iteration, we start with $\mathcal{G}=\Omega$ and $\mathcal{I}=\emptyset$, since we observe that the subset $\mathcal{G}$ usually constitues a major part in $\Omega$.

We propose an Iterative Joint Super Resolution (IJSR) algorithm, which decomposes (4) into three subproblems and solves them iteratively:

- Symmetric sparse coding: fix $\mathbf{X}$, update $\left\{\mathbf{a}_{i j}^{H}\right\},\left\{\mathbf{a}_{i j}^{L}\right\}$

- Component classification: fix $\mathbf{X},\left\{\mathbf{a}_{i j}^{H}\right\},\left\{\mathbf{a}_{i j}^{L}\right\}$, update $\mathcal{G}, \mathcal{I}$

- Latent image recovery: fix $\mathcal{G}, \mathcal{I},\left\{\mathbf{a}_{i j}^{H}\right\},\left\{\mathbf{a}_{i j}^{L}\right\}$, update $\mathrm{X}$

The estimated HR image in the $k$-th iteration, denoted as $\mathbf{X}^{\mathbf{k}}$, is updated iteratively. The initialization $\mathbf{X}^{\mathbf{0}}$ is upsampled from $\mathbf{Y}$ by bi-cubic interpolation. Such iterations are viewed as converged and terminated on condition that $\frac{\left\|\mathbf{X}^{k}-\mathbf{X}^{k-1}\right\|_{2}^{2}}{\left\|\mathbf{X}^{k}\right\|_{2}^{2}}<\epsilon$, where $\epsilon$ is a given threshold.

\subsection{Symmetric Sparse Coding}

We first update $\left\{\mathbf{a}_{i j}^{H}\right\},\left\{\mathbf{a}_{i j}^{L}\right\}$ by fixing $\mathbf{X}=\mathbf{X}^{\mathbf{k}-\mathbf{1}}$. Solving (4) is then reduced to minimizing the $\ell_{G}$ function over $\left\{\mathbf{a}_{i j}^{H}\right\},\left\{\mathbf{a}_{i j}^{L}\right\}$ only. The minimization is patch-wise separatable:

$$
\begin{array}{cc}
\min _{\mathbf{a}_{i j}^{H}, \mathbf{a}_{i j}^{L}} & \left(\left\|\mathbf{a}_{i j}^{H}\right\|_{1}+\left\|\mathbf{a}_{i j}^{L}\right\|_{1}\right)+\lambda_{l}\left\|D_{l} \mathbf{a}_{i j}^{L}-\mathbf{Y}_{i j}\right\|_{2}^{2}+ \\
& \lambda_{h}\left\|D_{h} \mathbf{a}_{i j}^{H}-\mathbf{X}_{\mathbf{i j}}^{\mathbf{k}-\mathbf{1}}\right\|_{2}^{2}+\theta\left\|\mathbf{a}_{i j}^{H}-\mathbf{a}_{i j}^{L}\right\|_{2}^{2}
\end{array}
$$

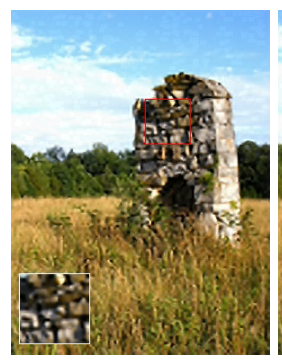

(a) Iteration 1

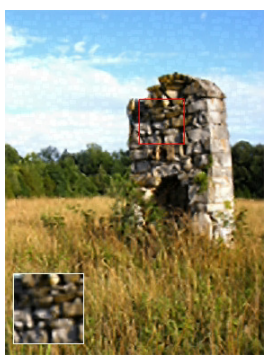

(b) Iteration 3

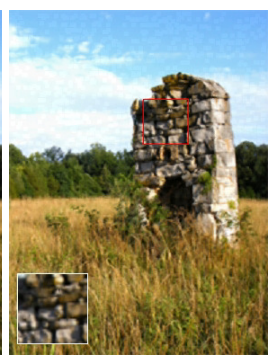

(c) Iteration 7
Figure 3. Intermediate results on the "Wall" image. Note the texture in the zoomed region becomes clearer as iteration goes.

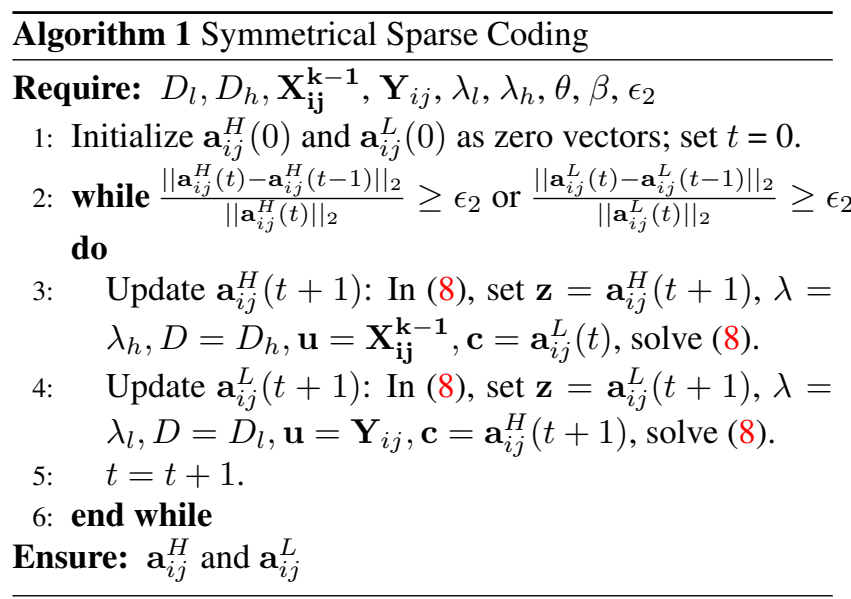

We then adopt the Alternating Direction Method (ADM) [14] to minimize (7) with respect to $\mathbf{a}_{i j}^{H}$ and $\mathbf{a}_{i j}^{L}$ in an alternating manner. With either $\mathbf{a}_{i j}^{H}$ or $\mathbf{a}_{i j}^{L}$ fixed, (7) will share the same minimization form:

$$
\min _{\mathbf{z}}\|\mathbf{z}\|_{1}+\lambda\|D \mathbf{z}-\mathbf{u}\|_{2}^{2}+\theta\|\mathbf{z}-\mathbf{c}\|_{2}^{2}
$$

(8) is a lasso-type problem and can be solved by algorithms introduced in [12]. Based on that, we can give the solution to (7) by Algorithm I.

\subsection{Component Classification}

The updating of $\mathcal{G}$ and $\mathcal{I}$ is fulfilled by implementing a classifier $\mathbf{T}\left(\mathbf{a}_{i j}^{H}, \mathbf{a}_{i j}^{L}\right)$, which needs to reflect whether a patch can be properly represented by the given coupled dictionaries. Empirical choices include: 1) the sparsity of the sparse representation $\mathbf{a}_{i j}^{L}$ (or $\mathbf{a}_{i j}^{H}$ ), i.e., $\left.\left\|\mathbf{a}_{i j}^{L}\right\|_{0} ; 2\right)$ the residual energy of sparse coding, i.e., $\left\|D_{l} \mathbf{a}_{i j}^{L}-\mathbf{Y}_{i j}\right\|_{2}^{2}$. As observed in extensive experiments, if a patch is poorly coded, either the representation $\mathbf{a}_{i j}^{L}$ tends to be extremely sparse or its residual energy will be large. Fig. 2 (a) shows the patch-level error map between the sparse coding SR result and the ground truth HR image $\mathbf{X}$ for "Wall" image. Fig. 2 (b) displays the sparsity map for $\left\|\mathbf{a}_{i j}^{L}\right\|_{0}$. Fig 2 (c) presents the residual map for $\left\|D_{l} \mathbf{a}_{i j}^{L}-\mathbf{Y}_{i j}\right\|_{2}^{2}$. It verifies that the 


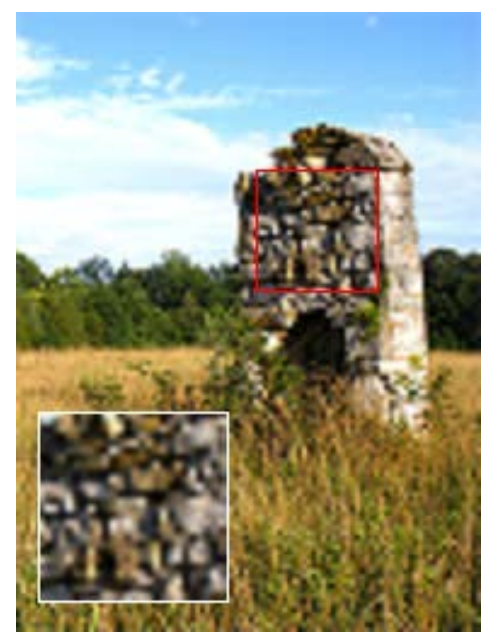

(a) Bi-cubic

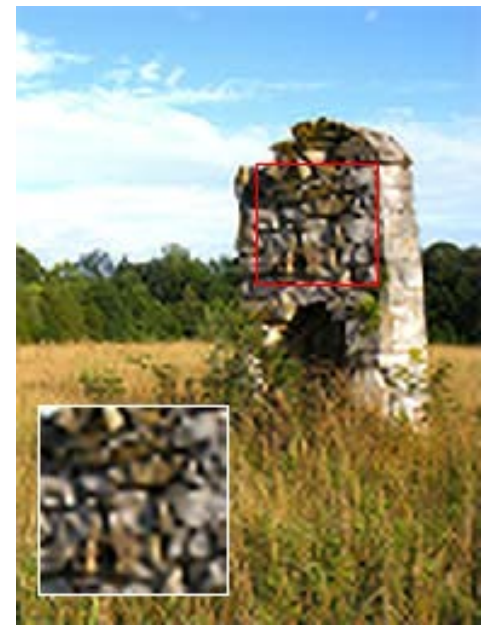

(b) Yang et al. [6]

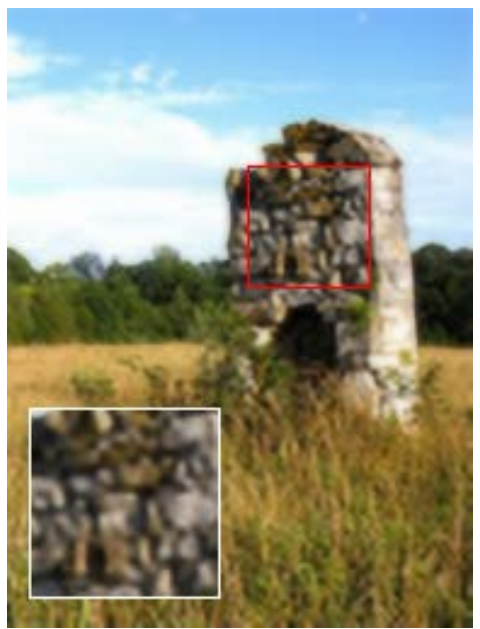

(c) Dong et al. [13]

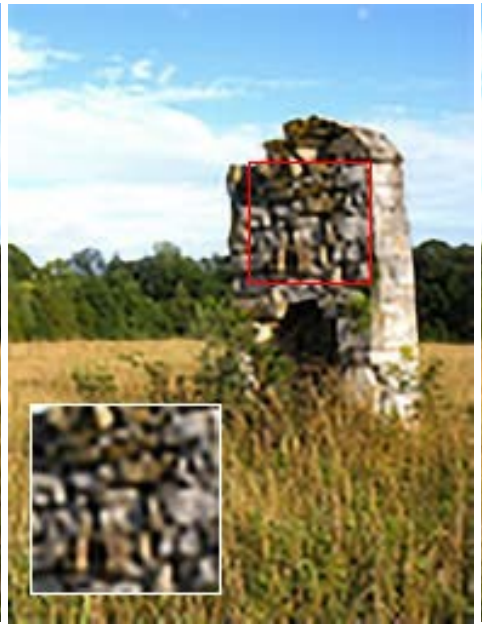

(d) Glasner et al. [7]

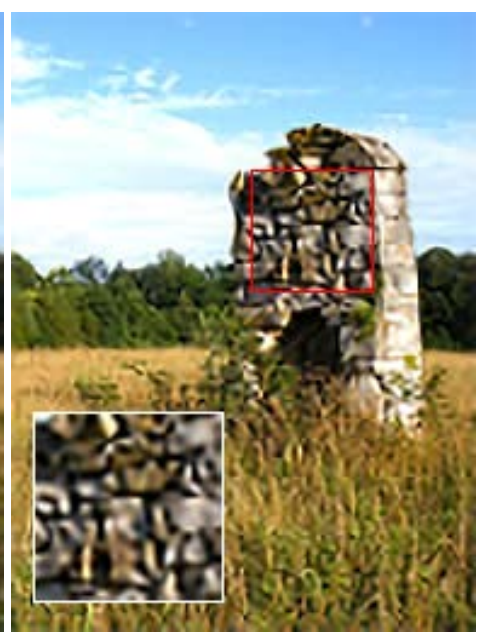

(e) Freedman and Fattal [8]

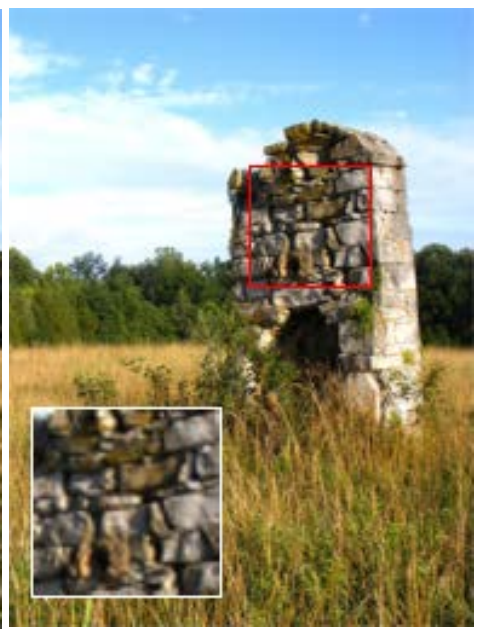

(f) Proposed

Figure 4. Visual comparisons among various SR methods on the "Wall" image.

patches with large errors or distortions under sparse coding are very likely to have both very sparse coefficients and large residual values.

Let $t_{1}$ and $t_{2}$ be the values of $\left\|\mathbf{a}_{i j}^{L}\right\|_{0}$ and $\left\|D_{l} \mathbf{a}_{i j}^{L}-\mathbf{Y}_{i j}\right\|_{2}^{2}$ linearly normalized between $[0,1]$. We define the indicator $\mathbf{T}\left(\mathbf{a}_{i j}^{H}, \mathbf{a}_{i j}^{L}\right)$ of the following form:

$$
\mathbf{T}\left(\mathbf{a}_{i j}^{H}, \mathbf{a}_{i j}^{L}\right)= \begin{cases}1 & \text { if }\left(1-t_{1}\right) \times t_{2} \leq \epsilon_{t} \\ 0 & \text { otherwise }\end{cases}
$$

A patch $\mathbf{X}_{i j}$ will be classified into $P_{\mathcal{G}}(\mathbf{X})$ if $\mathbf{T}\left(\mathbf{a}_{i j}^{H}, \mathbf{a}_{i j}^{L}\right)$ is 1 , and $P_{\mathcal{I}}(\mathbf{X})$ if $\mathbf{T}\left(\mathbf{a}_{i j}^{H}, \mathbf{a}_{i j}^{L}\right)$ is 0 . Fig. 2 (d) shows that the large values of our proposed indicator hold good correspondences to those regions where large estimation errors are introduced from the previous step. We can also see a good co-linearity from Fig. 2 (e), which manifests that the real errors and indicator values have a strong correlation, especially when the error turns large. The observation verifies the effectiveness of the proposed classifier.

\subsection{Latent Image Recovery}

The updating of $\mathbf{X}$ consists of two parts, i.e., the updating of $P_{\mathcal{G}}(\mathbf{X})$ and $P_{\mathcal{I}}(\mathbf{X})$. The former is trivial as we believe coupled sparse coding will have satisfactory performance over $P_{\mathcal{G}}(\mathbf{X})$, we simply let $\mathbf{X}_{\mathbf{i j}}^{\mathbf{k}}=D_{h} \mathbf{a}_{i j}^{H}$ for those patches where $\mathbf{T}\left(\mathbf{a}_{i j}^{H}, \mathbf{a}_{i j}^{L}\right)=1$. For patches in $P_{\mathcal{I}}(\mathbf{X})$, we first exploit the local scale invariance prior by solving (3). Then we work on (4) with $\mathcal{G}, \mathcal{I},\left\{\mathbf{a}_{i j}^{H}\right\}$ and $\left\{\mathbf{a}_{i j}^{L}\right\}$ fixed, which is equivalent to the following optimization:

$$
\begin{aligned}
\min _{\mathbf{X}_{\mathbf{i j}}^{\mathbf{k}}} & \alpha\left\|\mathbf{X}_{i j}^{\prime}+\mathbf{Y}_{m^{*} n^{*}}-\mathbf{Y}_{m^{*} n^{*}}^{\prime}-\mathbf{X}_{\mathbf{i j}}^{\mathbf{k}}\right\|_{2}^{2} \\
& +\lambda_{h}\left\|D_{h} \mathbf{a}_{i j}^{H}-\mathbf{X}_{\mathbf{i j}}^{\mathbf{k}}\right\|_{2}^{2}
\end{aligned}
$$

Eqn. (10) could be viewed as a further refinement to directly minimizing the $\ell_{I}(\cdot)$ function (6). In experiments we 


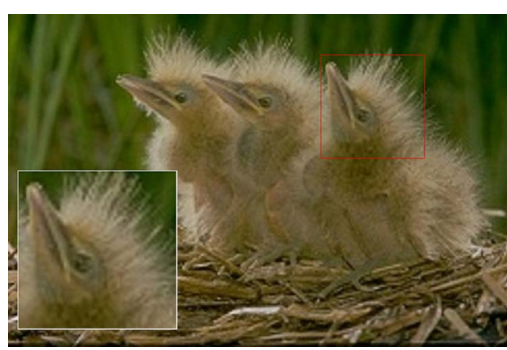

(a) Bi-cubic

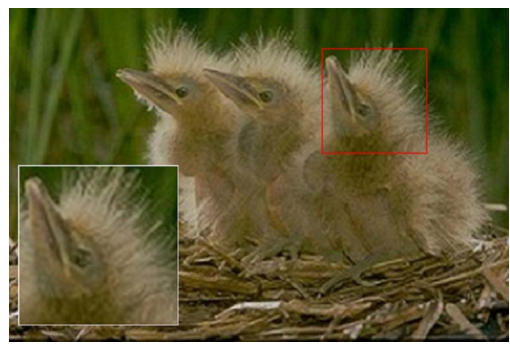

(b) Yang et al. [6]

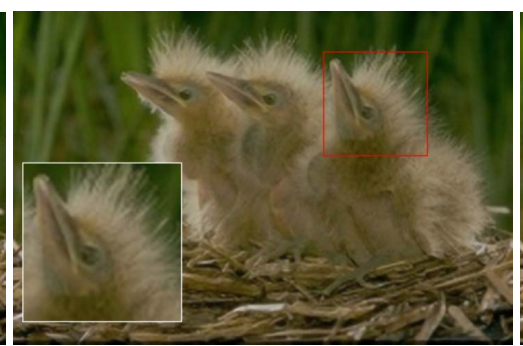

(c) Dong et al. [13]

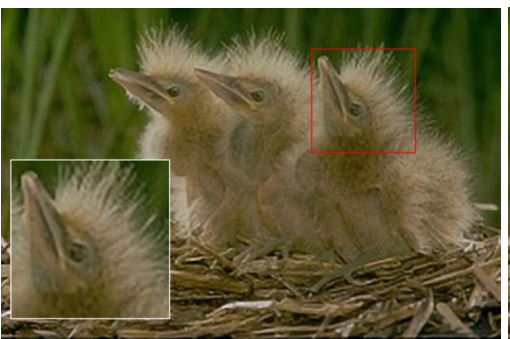

(d) Glasner et al. [7]

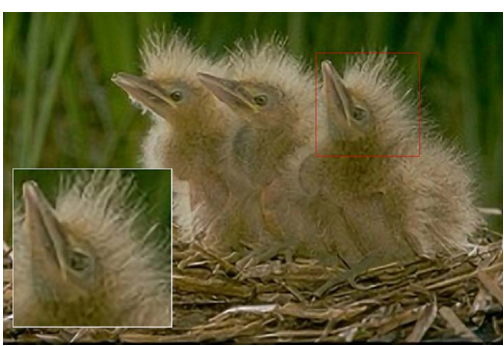

(e) Freedman and Fattal [8]

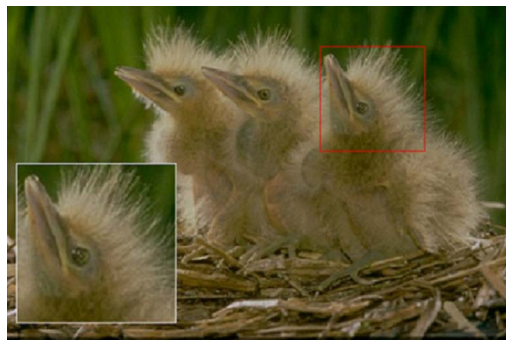

(f) Proposed

Figure 5. Visual comparisons among various SR methods on the "Nestling” image.

find such a relaxation helpful for suppressing the unnaturallooking artifacts, as $D_{h} \mathbf{a}_{i j}^{h}$ can also contain useful reference information even though the patch itself is judged as $P_{\mathcal{G}}(\mathbf{X})$ part. Eqn. (10) can be solved efficiently with a closed-form solution:

$$
\mathbf{X}_{\mathbf{i j}}^{\mathbf{k}}=\frac{\alpha\left(\mathbf{X}^{\prime}{ }_{i j}+\mathbf{Y}_{m^{*} n^{*}}-\mathbf{Y}_{m^{*} n^{*}}^{\prime}\right)+D_{h} \mathbf{a}_{i j}^{H}}{\alpha+\lambda_{h}}
$$

\section{Experiments}

\subsection{Implementation Details}

In our experiments, we magnify all the input LR images by a factor of 3 . We always use $5 \times 5$ LR patches with 1 pixel overlapping to ensure spatial consistency. For color images, we apply IJSR algorithm to the illuminance channel only, as humans are more sensitive to illuminance changes. We then interpolate the color layers $(\mathrm{Cb}, \mathrm{Cr})$ using plain bi-cubic interpolation.

The IJSR algorithm contains a set of parameters that influence the final SR results. In the following, we list the empirical parameter settings: For Eqn. (4), we set $\lambda_{l}=1$ and $\lambda_{h}=0.5$ to allow the LR patches to have slightly larger influences on deciding the final minimization results because LR patches are authentic. $\alpha$ is set to be $10 . \theta$ is fixed as 5 to strongly enforce the equivalence between $\mathbf{a}_{i j}^{H}$ and $\mathbf{a}_{i j}^{L}$. For component classification, in each iteration, the threshold $\epsilon_{T}$ is adaptively selected to ensure that patches with the top 10 percent largest indicator values will be classified into $I$. For latent image recovery, we use the same filter bank and multi-scale small factor series as presented in [8] to find $\left(m^{*}, n^{*}\right)$.

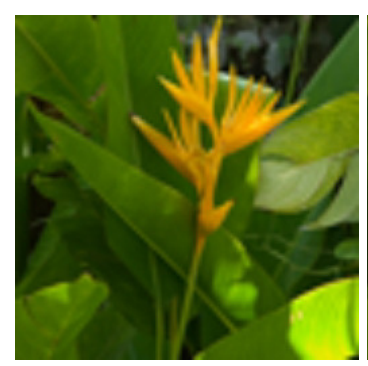

(a) Bi-cubic

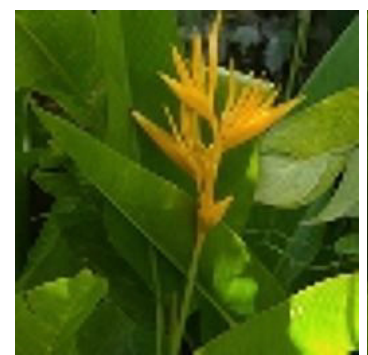

(c) Dong et al. [13]

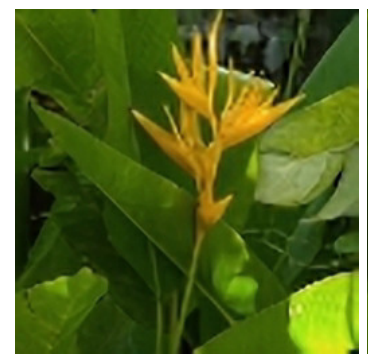

(e) Freedman and Fattal [8]

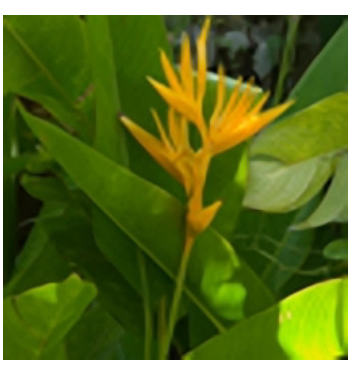

(b) Yang et al. [6]

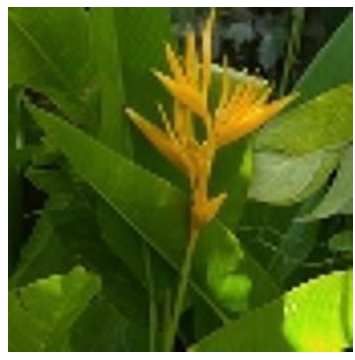

(d) Glasner et al. [7]

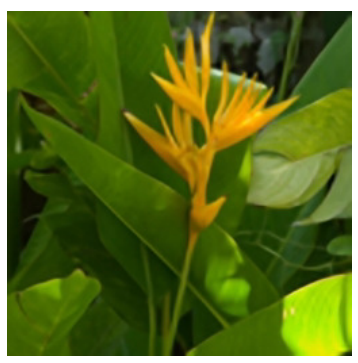

(f) Proposed
Figure 6. Visual comparisons among various SR methods on the "Plant" image. 
Table 1. PSNR and SSIM performance comparisons for five input images

\begin{tabular}{|c|c|c|c|c|c|c|c|}
\hline & & Bi-cubic & Yang et al. [6] & Dong et al. [13] & Glasner et al[7] & Freedman and Fattal [8] & Proposed \\
\hline \multirow{2}{*}{ Wall } & PSNR & 24.17 & 24.87 & 25.11 & 23.36 & 25.50 & $\mathbf{3 0 . 5 8}$ \\
\cline { 2 - 8 } & SSIM & 0.8456 & 0.8325 & 0.8102 & 0.8295 & 0.8550 & $\mathbf{0 . 9 2 0 3}$ \\
\hline \multirow{2}{*}{ Nestling } & PSNR & 26.54 & 28.51 & 28.77 & 26.93 & 28.93 & $\mathbf{3 0 . 8 1}$ \\
\cline { 2 - 8 } & SSIM & 0.8554 & 0.8951 & 0.8774 & 0.8871 & 0.8943 & $\mathbf{0 . 9 1 8 1}$ \\
\hline \multirow{2}{*}{ Plant } & PSNR & 30.34 & 32.55 & 30.99 & 30.33 & 32.75 & $\mathbf{3 4 . 9 3}$ \\
\cline { 2 - 8 } & SSIM & 0.8969 & 0.9284 & 0.9221 & 0.9087 & 0.9286 & $\mathbf{0 . 9 3 7 7}$ \\
\hline \multirow{2}{*}{ Parrot } & PSNR & 26.15 & 29.51 & 27.93 & 28.39 & 27.82 & $\mathbf{3 0 . 7 2}$ \\
\cline { 2 - 8 } & SSIM & 0.8991 & 0.9306 & 0.9143 & 0.9003 & 0.9237 & $\mathbf{0 . 9 4 8 9}$ \\
\hline \multirow{2}{*}{ Butterfly } & PSNR & 29.82 & 31.89 & 31.05 & 29.59 & 30.74 & $\mathbf{3 2 . 2 3}$ \\
\cline { 2 - 8 } & SSIM & 0.9182 & $\mathbf{0 . 9 4 7 8}$ & 0.9416 & 0.9305 & 0.9298 & 0.9456 \\
\hline
\end{tabular}

We did our best to present the parameter choices that give the overall optimal SR performance, but we acknowledge the possibility that better results may be obtained with finer parameter tuning. Because this paper mainly contributes to providing a joint insight into SR and the dictionary training part is not a current focus, we simply adopt the same $D_{l}$ and $D_{h}$ trained in [5] due to the similar roles played by the dictionaries in their formulation and our $\ell_{G}$ function. However, we are aware that such $D_{l}$ and $D_{h}$ are not optimized for the proposed method. It implies that the performance of our proposed method can be further promoted by integrating a specifically-designed dictionary learning part. We will leave it to future work.

\subsection{Intermediate Results during Iterations}

IJSR algorithm typically takes 5-10 iterations to converge under the above settings. The generic and individual priors interact with each other iteratively to improve SR quality. To help the readers better feel the procedure, in Fig. 3 we present the intermediate results of IJSR algorithm at iteration 1, 3, 7, when applied to "Wall" image (total iteration number is 10). More detailed comparisons can be observed from the zoomed regions.

\subsection{Comparison with State-of-the-art Results}

\subsubsection{Quantitative Comparison}

We compare the proposed method with bi-cubic interpolation (as a baseline), two state-of-the-art sparse coding-based methods [6] and [13], two state-of-the-art self-similarity based methods [7] and [8]. In our experiments, we produce SR results by using the original codes of $[6,13,7]$, and implement the algorithm in [8]. We make assessments for the qualities of SR results by two quantitative measurements, i.e., peak signal-to-noise ratio (PSNR), and the structure similarity (SSIM) of SR result and groundtruth index between $[0,1]$ (closer to 1 means higher similarity). We list PSNR and SSIM values for five test images by different methods in Table 1. On all test images, our algorithm outperforms others significantly in terms of PSNR. In all cases except the "Butterfly" test image, our algorithm achieves much higher SSIM scores than other methods. These results clearly demonstrate the superiority of our proposed approach.

\subsubsection{Qualitative Comparison}

As the visual quality is typically the most important criterion for single image SR, here we list the visual comparison results for all the test images (results best viewed on a highresolution display). Detail comparisons can be found at the enlarged local regions.

In Fig. 4, the "Wall" image is very challenging for SR since it is abundant in complex structures (the brick wall) and various kinds of fine textures (grass, forest, etc.). Although outperforming the bi-cubic interpolation a lot in preserving details, Yang et. al's method brings a few ringings and jaggies to the brick regions, as well as the grass region. Dong et. al's method, though presenting less jaggy artifacts than Yang et. al's, leads to oversmoothing of the wall structure. Glasner et. al's and Freedman and Fattal's method are able to give sharper results, but the edges of bricks look rigid and artificial. Our proposed method produces an impressive visually-pleasant SR result, and meanwhile preserves faithful structures as well as textural details. In Fig. 5 , whereas the results of all prior methods are still unsatisfactory, our proposed method produces a both artifact-free and detail-preserving SR result. The feathers are naturallooking and detail-preserving. It is also noteworthy that our method reconstruct a much better eye part than all the others. More comparison results could be found in Fig. 6-8 as well. Our results are consistently sharper than Yang et. al's and Dong et. al's, and are free of artifacts observed on those of Glasner et al.'s and Freedman and Fattal's. 


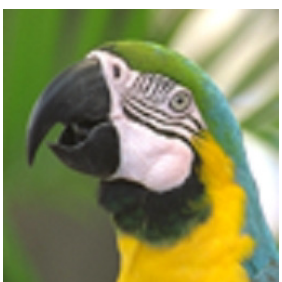

(a) Bi-cubic

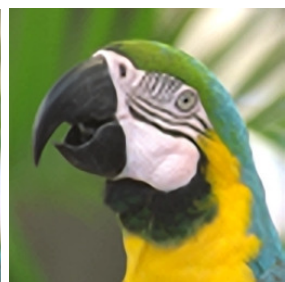

(b) Yang et al. [6]

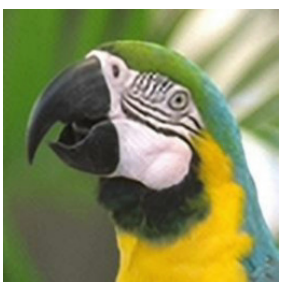

(c) Dong et al. [13]

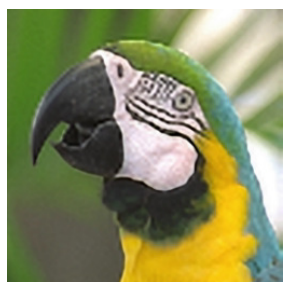

(d) Glasner et al. [7]

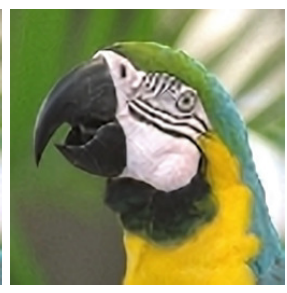

(e) Freedman and Fattal [8]

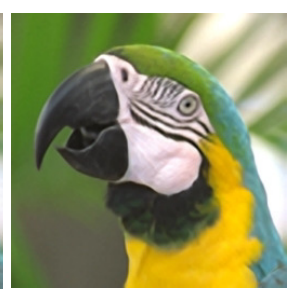

(f) Proposed

Figure 7. Visual comparisons among various SR methods on the "Parrot" image.

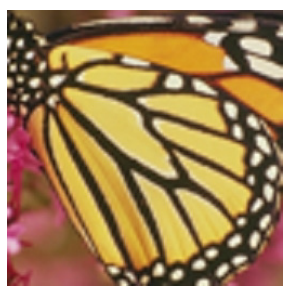

(a) Bi-cubic

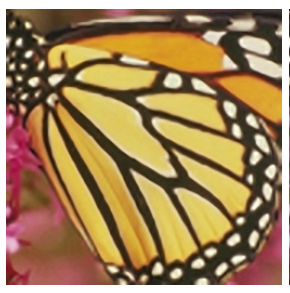

(b) Yang et al. [6]

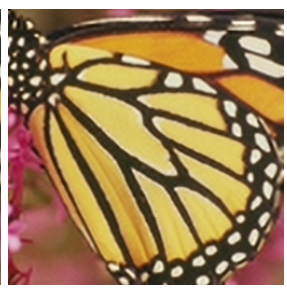

(c) Dong et al. [13]

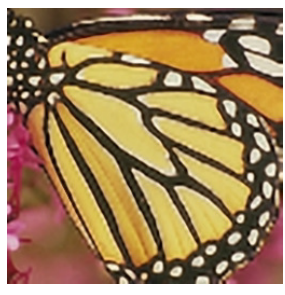

(d) Glasner et al. [7]

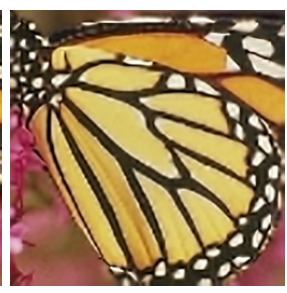

(e) Freedman and Fattal [8]

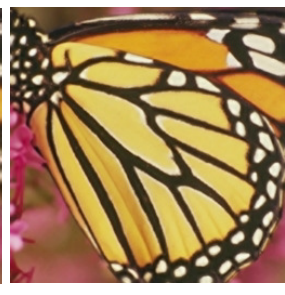

(f) Proposed

Figure 8. Visual comparisons among various SR methods on the "Butterfly" image.

\section{Conclusion}

This paper presents a novel joint perspective towards single image SR. We validate our viewpoint by analyzing the complementary properties of external- and self-example based methods, and exploit priors from both perspectives. A unified joint formulation is proposed based on such insights, and an IJSR algorithm is designed to give its solution. Experimental results demonstrate the clear effectiveness of the unified framework in terms of both quantitative and qualitative comparisons. Our future work will integrate dictionary learning into the proposed scheme for further performance improvement. We are also interested in pursuing an adaptive parameter adjustment strategy for IJSR algorithm, as well as reducing its complexity.

\section{References}

[1] S. C. Park, M. K. Park, K. G. Kang, "Super-Resolution Image Reconstruction: A Technical Overview," IEEE Signal Processing Magazine, vol. 20, no. 3, pp. 21-36, 2003. 1

[2] B. Morse, D. Schwartzwald, "Image Magnification Using Level Set Reconstruction," In Proceedings of IEEE Conference on Computer Vision and Pattern Recognition (CVPR), pp 333-341, (2001)

[3] R. Fattal, "Image Upsampling via Imposed Edge Statistics," ACM Transactions on Graphics, vol. 26, no. 3, 20071

[4] Z. Lin, H. Y. Shum, "Fundamental Limits of Reconstructionbased Superresolution Algorithms Under Local Translation," IEEE Transactions on Pattern Analysis and Machine Intelligence, vol. 26, no. 1, pp. 83-97, 20041

[5] J. Yang, J. Wright, T. Huang and Y. Ma, "Image Superresolution as Sparse Representation of Raw Image Patches," In Proceeding of IEEE Conference on Computer Vision and Pattern Recognition (CVPR), 2008. 1
[6] J. Yang, J. Wright, T. Huang and Y. Ma, "Image Superresolution via Sparse Representation," IEEE Transactions on Image Processing, vol. 19, no. 11, pp. 2861-2873, 2010. 1, 2, 7

[7] J. Yang, Z. Wang, Z. Lin, S. Chen and T. Huang, "Coupled Dictionary Training for Image Super-resolution," IEEE Transactions on Image Processing, vol. 21, no. 8, pp. 34673478, 2012. 1, 2, 5, 6, 7, 8

[8] D. Glasner, S. Bagon and M. Irani, "Super-resolution from a single image" in Proceeding of IEEE International Conference on Computer Vision (ICCV), 2009. 2, 5, 6, 7, 8

[9] G. Freedman and R. Fattal, "Image and Video Upscaling from Local Self-Examples," ACM Transactions on Graphics, vol. 28, no. 3, 2010 1, 2, 3, 5, 6, 7, 8

[10] C. Yang, J. Huang, and M. Yang, "Exploiting SelfSimilarities for Single Frame Super-Resolution" In Proceedings of Asian Conference on Computer Vision (ACCV), pp. 1807-1818, 2010. 1, 2

[11] M. Zontak, "Internal Statistics of A Single Natural Image," In Proceedings of IEEE International Conference on Computer Vision (ICCV), pp. 977-984, 20112

[12] J. Mairal, F. Bach, J. Ponce, G. Sapiro and A. Zisserman, "Non-local Sparse Models for Image Restoration," In Proceedings of IEEE International Conference on Computer Vision (ICCV), pp. 2272-2279, 20092

[13] H. Lee, A. Battle, R. Raina and A. Y. Ng, "Efficient Sparse Coding Algorithms," In Proceedings of Neural Information Processing Systems (NIPS), pp. 801-808, 2007. 2, 4

[14] W. Dong, L. Zhang, G. Shi and X. Li, "Nonlocally Centralized Sparse Representation for Image Restoration,” IEEE Transactions on Image Processing, vol. 22, no. 4, pp. 16201630, 2012. 2, 5, 6, 7, 8

[15] D. Bertsekas, Nonlinear Programming, Athena Scientific, Second Edition, 1999. 4 